\title{
Vibration fatigue at half-sine impulse excitation in the time and frequency domains
}

\author{
Primož Ogrinec ${ }^{a}$, Janko Slavič ${ }^{a, 1},{\text { Martin } \check{C ̆ e s n i k}^{a} \text {, Miha Boltežar }}^{a}$ \\ ${ }^{a}$ University of Ljubljana, Faculty of Mechanical Engineering, Aškerčeva c. 6, 1000 \\ Ljubljana, Slovenia \\ Cite as: \\ P. Ogrinec, J. Slavič, M. Česnik, M. Boltežar. Vibration fatigue at half-sine impulse \\ excitation in the time and frequency domains. International Journal of Fatigue, \\ Volume 123, June 2019, Pages 308-317, doi.org/10.1016/j.ijfatigue.2019.02.031
}

\begin{abstract}
Three types of loads are typical in vibration fatigue: random, harmonic and impact. In an application, any combination of these loads is possible. In vibration fatigue the random loads can be investigated by using the frequencyresponse function of the structure. While the theoretical relationship between the frequency-response function and the impact-response function is clear (they form the Fourier pair), the time/frequency relationship of vibration-fatigue damage has not yet been investigated in detail. The focus of this research is a theoretical study of the time- and frequency-domain damage of a single-degreeof-freedom dynamic system under well-separated half-sine impulse excitation. The introduced theoretical relationship between the time- and frequency-domain (Narrow-band and Tovo-Benasciutti method) damage showed that the significantly different results are related to the underlying dynamic properties (e.g., natural frequency and damping) of a flexible system. Based on the introduced relationship, the frequency-domain damage estimation is corrected for a reliable damage estimation. In addition, an experimental test case is presented. The introduced theoretical relationship opens up new possibilities to investigate the impact excitation in the frequency domain and provides the necessary theoret-
\end{abstract}

\footnotetext{
${ }^{1}$ Corresponding author. Tel.: +386 14771 226. Email address: janko.slavic@fs.uni-lj.si
} 
ical background for applications when the impacts are not well separated and start to overlap, resulting in the broadband excitation observed in clearance contacts.

Keywords: vibration fatigue, random loads, spectral methods, fatigue life, stationary and non-stationary loading, non-Gaussian loading

\section{Introduction}

Flexible structures subject to vibrations experience material fatigue, often referred to as vibration fatigue. In most cases the excitation vibrations are of a random nature, harmonic or impulse. Harmonic and impulse loads are

5 deterministic and can be described analytically for linear systems in the time and frequency domains. Random loads are stochastic, and a frequency-domain analysis is possible using the assumptions of linearity, stationarity and Gaussianity [1. In an application, the structures are exposed to a combination of harmonic, random and impulse loads [2, 3]. While a combination of harmonic and random loads can be studied in the frequency domain [4, 5, 6, 7], the relationship of an impulse excitation in the time and frequency domains is not well researched. These impulse loads can originate in increased clearances in contacts due to wear, unforeseen use of the component, various loading conditions, etc. The presence of these impulses can significantly influence the frequency domain fatigue life predictions [8], even when dealing with loads well within the linear region. In the design phase of a component, the requirements for load optimised structures and reduction of mass can significantly influence the structural dynamics of the component and subsequently their fatigue life. Therefore an accurate fatigue life assessment is crucial for definitive conclusions in the development of the structure.

Fatigue life is typically estimated using counting methods in the time domain (e.g., rainflow counting method [9]), or in the frequency domain (e.g., Narrow band[10, Dirlik [11] or Tovo-Benasciutti method [12]). The impulse response of a linear single-degree-of-freedom system is deterministic and a time-domain 
analysis would be preferable. However, when the impulses are randomly spaced, when the time histories are long, or where the impulse loads are combined with other load types, a frequency-domain analysis of the damage is prefered. Frequency-domain methods mostly rely on the assumption of a Gaussian stationary signal [1, 8, 13]. The presence of impulse signals renders the excitation

so signal non-stationary and non-Gaussian, and thus calls for further research of counting methods in the frequency domain.

Recently, a great deal of effort was made to develop the frequency-counting methods for the analysis of vibrationaly excited structures with non-Gaussian and non-stationary excitation signals [14, 15. Benasciutti and Tovo [12 re35 searched the non-stationary switching random loads.

Ciantetti et al. [16] researched the damage rate from non-Gaussian signals using a correction factor. Wolfsteiner [17, 18, 19] showed that a long signal can be decomposed into Gaussian portions and analyzed with existing frequencydomain methods. Similarly, an empirical decomposition model was derived by 40 Niu et al. in 20]. Rizzi et al. 21] showed that when dealing with non-Gaussian excitation signals, where the rate of impulses is much greater than the time of the system's impulse response, the response of a linear system is Gaussian and frequency-domain counting methods can be applied. The lumping-block equivalence method, where the non-stationary loads are divided into stationary 45 sections, was studied in 22 .

Palmieri et al. [8] experimentally and theoretically researched the importance of non-gaussianity and non-stationarity in vibrational fatigue and found that the latter is significantly more important. Capponi et al. 23 introduced an index of non-stationarity that quantifies the deviation from the Gaussian 50 stationary signals.

The main goal of this study is to analyze the use of standard vibrationfatigue methods [11, 24] when dealing with high-cycle impulse excitation on a single-degree-of-freedom structure. The system response is analyzed numerically and the influence of the damping ratio and the natural frequency on the error ${ }_{55}$ of the frequency-counting method in relation to the rainflow-counting method 
is studied. An experimental setup is used to confirm the introduced theoretical relationships.

This manuscript is organized as follows. Section 2 presents the theoretical background. In Section 3 the impulse excitation is theoretically investigated using the time domain (rainflow-counting method) and the frequency domain (narrow-band [10] and Tovo-Bennasciutti [12] method). The comparison is prepared for a singe-degree-of-freedom (SDOF) system, which in structural dynamics theory is relatively easily expanded to the arbitrary multiple-degreeof-freedom (MDOF) case. The effect of damping and natural frequency on the error of the frequency method is analyzed, and the ratio between timeand frequency-domain methods is derived. In Section 4 an experimental setup is presented that confirms the introduced theoretical time/frequency relations. Section 5 draws the conclusions.

\section{Theoretical background}

\subsection{Structural dynamics and impulse response}

The fundamentals of structural dynamics are explained in more detail in Appendix A.

When a single degree of freedom (SDOF) system [25] is excited with an ideal unit impulse, this is defined with the Dirac delta function:

$$
\delta(t)= \begin{cases}\infty, & t=0 \\ 0, & t \neq 0\end{cases}
$$

the systems responds with the impulse response function $g(t)$ [26, 25]:

$$
g(t)=\frac{\mathrm{e}^{-\delta \omega_{0} t} \sin \left(\sqrt{1-\delta^{2}} \omega_{0} t\right)}{\sqrt{1-\delta^{2}} \omega_{0}} .
$$

When the damping coefficient is small $\left(\delta<<1, \sqrt{1-\delta^{2}} \approx 1\right)$ then Eq. (2) simplifies to:

$$
g(t)=\frac{\mathrm{e}^{-\delta \omega_{0} t} \sin \left(\omega_{0} t\right)}{\omega_{0}} .
$$


In order to obtain the response for a general force excitation $f(t)$, the convolution integral of the impulse $f(t)$ and the impulse-response function $g(t)$ are required [27.

$$
x(t)=f(t) * g(t)=\int_{-\infty}^{t} f(\tau) g(t-\tau) d \tau,
$$

where $t$ is the time variable and $x(t)$ is the time response of the SDOF system.

To obtain the response of the structure in the frequency domain, the Eq. (4) must be transformed into the frequency domain. The Fourier transformation of the convolution integral in the time domain is the multiplication of the Fourier transforms of the two time-domain signals $f$ and $g$ [28]:

$$
X(\omega)=\mathfrak{F}\{f(t) * g(t)\}=F(\omega) G(\omega) .
$$

where the Fourier transform is:

$$
X(\omega)=\mathfrak{F}\{x(t)\}=\int_{-\infty}^{\infty} x(t) e^{2 \pi \mathrm{i} \omega t} \mathrm{~d} t .
$$

\subsection{The time-domain approach to a fatigue-life estimation}

Here, the basics (used later in this paper) of the time-domain approach to the fatigue-life estimation are presented (for details see, e.g., [29]).

The rainflow algorithm [9] transforms the stress time history into a series of stress reversals. After the stress time history is determined, the Miners rule of damage accumulation is applied and the damage is calculated as:

$$
D^{R F}=\sum_{i=1}^{M} \frac{n_{i}}{N_{i}}
$$

where $M$ is the number of ranges in the stress-reversal series that are identified by the rainflow algorithm and $n_{i}$ is the number of stress reversals at each individual stress level. Together with the fatigue parameters, which determine the expected number of cycles $N_{i}$ at a stress level, Eq. (7) leads to the accumulated damage in the structure. The number of expected cycles $N_{i}$ that a structure survives at the stress level $\sigma_{i}$ is theoretically described with the Wöhler curve 30]:

$$
\sigma=C N^{k}
$$


where $k$ and $C$ are the material's fatigue parameters. One has to be aware that this simple S-N relationship does not account for the material endurance limit and can only be used when dealing with high cycle fatigue [31.

\subsection{The frequency-domain approach to a fatigue-life estimation}

The basis of frequency-counting methods is the stress power spectrum density $S_{x x}(\omega)$, which is defined using Eq. (5):

$$
S_{x x}(\omega)=X^{*}(\omega) X(\omega),
$$

where the $X(\omega)$ is the complex amplitude spectrum and $X^{*}(\omega)$ its conjugate.

so There are several approaches to the time-to-failure estimations in the frequency domain [4, 32]. This research will be limited to the narrow-band method [10] and the Tovo-Bennasciutti method 24]. For other methods and their comparison see Mršnik et al. [4].

\subsubsection{The narrow-band method}

The narrow-band process is one where the spectral components of the excitation forces are expected to fall within a narrow frequency band, typically a width of $1 / 3$ of an octave band or less [33. When a process can be assumed to be in a narrow band, every peak coincides with a cycle. Therefore, the cycle's amplitudes are Rayleigh distributed. The narrow-band method for fatigue-damage estimation was presented by Miles [10]. For stress amplitudes the definition of the fatigue damage is 4 :

$$
D^{N B}=\nu_{0} C^{-1}\left(\sqrt{2} m_{0}\right)^{k} \Gamma\left(1+\frac{k}{2}\right),
$$

where $\Gamma(z)$ is the Euler Gamma function:

$$
\Gamma(z)=\int_{0}^{\infty} t^{z-1} \mathrm{e}^{-t} \mathrm{~d} t,
$$

$\nu_{0}$ is the expected positive zero-crossing intensity,

$$
\nu_{0}=\sqrt{\frac{m_{2}}{m_{0}}},
$$


$C$ and $k$ are the material's fatigue parameters, $m_{0}$ and $m_{2}$ are the 0 -th and 2-nd spectral moment respectively and $\alpha_{i}$ is determined as:

$$
\alpha_{i}=\frac{m_{i}}{\sqrt{m_{0} m_{2 i}}} .
$$

The spectral moment $m_{i}$ is defined as:

$$
m_{i}=\int_{0}^{\infty} \omega^{i} G_{x x}(\omega) \mathrm{d} \omega .
$$

where $G_{x x}(\omega)$ is the one-sided power spectral density $\left(2 S_{x x}\right.$ for $\omega>0$ and 0 for $\omega<0)[4]$.

\subsubsection{Tovo-Bennasciutti method}

The Tovo-Bennasciutti method has proven to be the most generally applicable frequency-domain method [4]. The impulse response of a SDOF structure can be regarded as a narrow-band process. However here, the Tovo-Bennasciutti method is being refered to as a more general frequency-domain method. For impulses that are superimposed on a broad-band random process, such a method would be required. With the Tovo-Bennasciutti method [12, 24] the fatigue-life can be calculated as a linear combination of the upper and lower fatigue-damage intensity limits, with the expression:

$$
D^{T B}=b+(1-b) \alpha_{2}^{k-1} \alpha_{2} D^{N B}
$$

where $b$ is:

$$
b=\min \left[1, \frac{\alpha_{1}-\alpha_{2}}{1-\alpha_{1}}\right],
$$

where $\alpha_{i}$ is defined with Eq. (13).

\subsection{Vibration fatigue}

Vibration fatigue relates the structural dynamics and the frequency-domain approach to a fatigue-life estimation.

The decoupled modal superposition approach A.2 provides the relationship between the excitation $\mathbf{F}$ and the response $\mathbf{X}$ :

$$
\mathbf{X}=\mathbf{H}(\omega) \mathbf{F},
$$


where $\mathbf{H}(\omega)$ is the receptance matrix [25. The $j k$-th element (where $j$ is the response and $k$ is the excitation index) of the receptance matrix is:

$$
H_{j k}(\omega)=\sum_{r=1}^{N} \frac{\Phi_{j r} \Phi_{k r}}{\omega_{r}^{2}-\omega^{2}+\mathrm{i} \eta_{r} \omega_{r}},
$$

where $\omega_{r}$ is the $r$-th natural frequency, $\eta_{r}$ is the modal damping for the $r$-th natural frequency and $\Phi_{j r}$ is the $j r$-th element of the mass-normalized modal matrix $\Phi$ [25]. However, for the fatigue-life estimation the stress-response tensor is required. The transfer-function matrices $\mathbf{H}_{\text {as }}$ present the relationship between the kinematic random excitation PSD $\mathbf{S}_{\mathbf{X}}(\omega)$ and the stress responses for the purpose of the fatigue analysis:

$$
\begin{gathered}
\mathbf{S}_{\mathbf{S}}(\omega)=\mathbf{H}_{\mathrm{as}}^{*}(\omega) \mathbf{H}_{\mathrm{as}}(\omega) \mathbf{S}_{\mathbf{X}}(\omega) . \\
\mathbf{S}_{\mathbf{S}}(\omega)=\left[\begin{array}{ccc}
S_{x x, x x}(\omega) & \ldots & S_{x x, y z}(\omega) \\
\ldots & \ddots & \ldots \\
S_{y z, x x}(\omega) & \ldots & S_{y z, y z}(\omega)
\end{array}\right] .
\end{gathered}
$$

The stress tensor $\mathbf{S}_{\mathbf{S}}(\omega)$ has 6 independent components. A common method is to determine an equivalent uniaxial stress state 34]. Other methods are described in [35. The equivalent stress is determined by:

$$
S_{e q}(\omega)=\mathbf{a} \mathbf{S}_{\mathbf{S}}(\omega) \mathbf{a}^{T},
$$

where $\mathbf{a}$ is a vector of coefficients for the used multi-axial criterion. For more details about the equivalent stress criteria see Niesłony and Macha 35] or Mršnik et al. 34.

Multi-axial loads are not researched here, so the equivalent stress criterion is not required. This manuscript focuses on SDOF systems, as the SDOF solution can be used on more complex systems via decoupled modal coordinates, Eq. A.3. The stress response 22 can be obtained by multiplying the kinematic response by a scalar coefficient $k_{\sigma} . k_{\sigma}$ can be determined experimentally.

$$
\sigma(t)=k_{\sigma} x(t)
$$




\section{Half-sine impulse excitation fatigue}

In accelerated vibration testing many forms of impulses are used [36], e.g., half-sine, rectangular, triangular, trapezoidal, haversine and sawtooth impulse [37. Here, the frequently used half-sine form will be researched in detail; it is defined as:

$$
f_{s / 2}(t)=\mathrm{A}(\theta(t)-\theta(t-\tau)) \sin \left(\omega_{0} t\right),
$$

where $\tau$ is the duration of the half-sine impulse, $A$ is the amplitude of the impulse, and $\theta(t)$ is the Heaviside step function. The normalized half-sine impulse is presented in Fig. 1

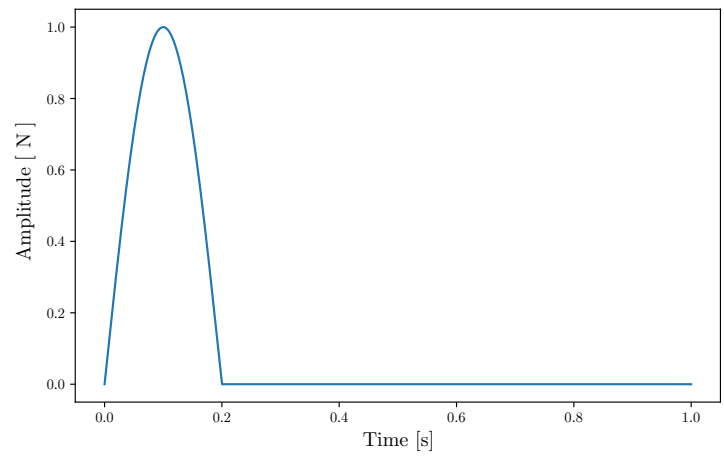

Figure 1: Normalized half-sine impulse.

If the duration of the impulse is too long the natural response at $\omega_{0}$ might not be excited [28. For this reason the half-sine impulse is related to the natural frequency $\omega_{0}$ of the excited SDOF structure:

$$
\omega_{0}=\frac{\pi}{a \tau},
$$

where $a>1$ is a constant. In this research $a=2$ will be used.

\subsection{SDOF response in the time domain}

In order to be able to compare the frequency domain to the time-domain approach, a counting method must be applied in the time and frequency do- 
stress response (4). As the stress $\sigma(t)$ is related to the kinematic response $x(t)$ using a linear scaling coefficient $k_{\sigma}(22)$, here the kinematic response $x(t)$ will be researched, instead of the stress response (4).

Due to the deterministic nature of the SDOF response to a half-sine impulse, the stress reversals can be obtained analytically. A cycle $\chi$ is the stress range between the two extremes of the SDOF system's response.

In order to determine the stress peaks the convolution integral

$$
x(t)=f_{s / 2}(t) * g(t)
$$

must be solved. However, as the response has the same rate of decline and frequency as the impulse response function $g(t)$, the latter can be used to identify the stress peaks, neglecting the transient phenomenon that does not generate a fatigue cycle for small damping ratios $(\delta<<1)$, Fig. 2 For this the impulseresponse function must be scaled to the amplitude of the impulse response, determined by the convolution integral Eq. (4).

The extremes of the load (see Fig. 2), assuming a small damping ratio $(\delta<<1)$, are:

$$
p_{n}=A_{c} g\left(\frac{(2 n-1) T_{0}}{4}\right) \quad n=1,2,3, \ldots
$$

where $T_{0}=2 \pi / \omega_{0}$ and $A_{c}$ is the amplitude of the response.

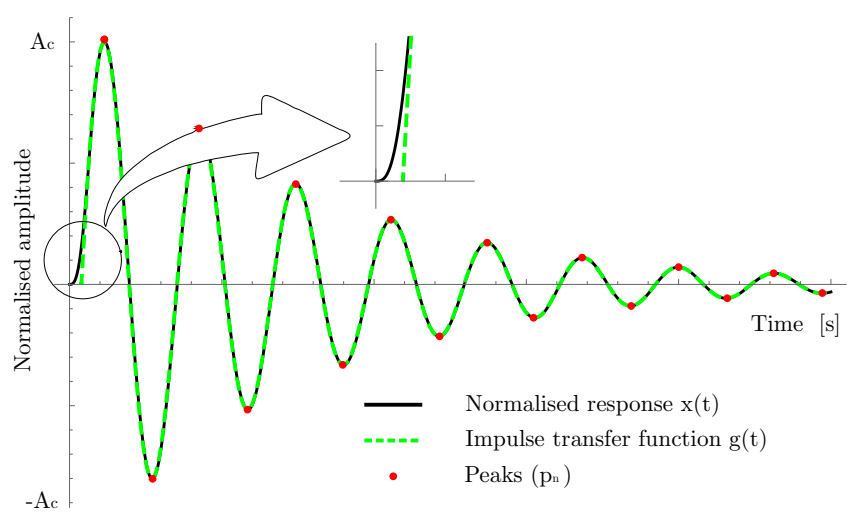

Figure 2: Comparison of the normalized stress response $x(t)$ and the impulse transfer function $g(t)$ with the corresponding peaks $p_{n}$. 
The amplitude of the response $A_{c}$ can be obtained by solving the convolution integral 25 and determining its maximum by finding the zero of its first derivative:

$$
\frac{\mathrm{d} x(t)}{\mathrm{d} t}=0
$$

The significant extreme occurs at:

$$
t_{0}=\frac{\cos ^{-1}\left(\frac{\left(a^{2}+1\right) \delta+\mathrm{e}^{\frac{\pi \delta}{a}}\left(\left(a^{2}+1\right) \delta \cos \left(\frac{\pi}{a}\right)-\left(a^{2}-1\right) \sin \left(\frac{\pi}{a}\right)\right)}{\sqrt{\left(a^{2}-1\right)^{2}\left(\mathrm{e}^{\frac{2 \pi \delta}{a}}+2 \mathrm{e}^{\frac{\pi \delta}{a}} \cos \left(\frac{\pi}{a}\right)+1\right)}}\right)}{\omega_{0}},
$$

and has the value:

$$
\begin{aligned}
A_{c}= & \frac{1}{\left(a^{2}-1\right)^{2} \omega_{0}^{2}}\left[a A \mathrm { e } ^ { \delta ( - t _ { 0 } ) \omega _ { 0 } } \left(\mathrm { e } ^ { \frac { \pi \delta } { a } } \left(2 \delta \cos \left(\frac{\pi}{a}-t_{0} \omega_{0}\right)-\right.\right.\right. \\
& \left.\left.\left.\left(a^{2}-1\right) \sin \left(\frac{\pi}{a}-t_{0} \omega_{0}\right)\right)+\left(a^{2}-1\right) \sin \left(t_{0} \omega_{0}\right)+2 \delta \cos \left(t_{0} \omega_{0}\right)\right)\right]
\end{aligned}
$$

Now that the amplitude of the response is known, the stress reversals must be

determined in order to determine the fatigue damage of the impulse response with the rainflow algorithm.

The stress reversal $\chi_{n}$ is the amplitude between the two extremes of the stress response:

$$
\chi_{n}=(-1)^{n+1} p_{n+1}+(-1)^{n} p_{n}, \quad n=1,2,3, \ldots
$$

Using Eq. (26) the stress reversals are:

$$
\chi_{n}=A_{c}(-1)^{n} \mathrm{e}^{-2 \pi \delta n}\left(\mathrm{e}^{\frac{1}{2} \pi \delta(2 n-1)}+\mathrm{e}^{\frac{1}{2} \pi \delta(2 n+1)}\right) \cos (\pi n),
$$

Taking into account that $n$ is an integer, Eq. 31 can be further simplified to:

$$
\chi_{n}=2 A_{c} \cosh \left(\frac{\pi \delta}{2}\right) \mathrm{e}^{\pi \delta(-n)} n=1,2,3, \ldots
$$

The first stress cycle $\chi_{0}$ is between 0 and the maximum amplitude of the stress response:

$$
\chi_{0}=A_{c} \frac{\mathrm{e}^{-\frac{1}{2}(\pi \delta)}}{2}
$$


Using Eq. (33) and 32 in the Palmgren-Miners rule of damage accumulation, Eq. (7), the damage of one impulse response is:

$$
D_{R}=\left(\frac{\chi_{0}}{2 C}\right)^{k}+\sum_{n=1}^{\infty}\left(\frac{\chi_{n}}{2 C}\right)^{k}
$$

where $k$ and $C$ are the material's fatigue properties.

The Cauchys criterion for convergence [38] states that for each $\epsilon>0$ a pair $k>n$ exists for which:

$$
\left|\sum_{j=n+1}^{r} \chi_{n}\right|=\left|\chi_{n+1}+\chi_{n+2}+\cdots+\chi_{r}\right|<\epsilon .
$$

Since the stress cycles of a damped SDOF structure limit towards $0 \lim _{n \rightarrow \infty} \chi_{n}=0$, the Cauchys criterion is satisfied and the sum in Eq. (34) is final:

$$
\sum_{n=1}^{\infty}\left(\frac{\chi_{n}}{2 C}\right)^{k}=\sum_{n=1}^{\infty}\left(\frac{(-1)^{n+1} p_{n+1}+(-1)^{n} p_{n}}{2 C}\right)^{k}=\frac{\left(\frac{A_{c} e^{-\frac{1}{2}(3 \pi \delta)} \cosh (\pi \delta / 2)\left(\mathrm{e}^{\pi \delta}+1\right)}{C \omega_{0}}\right)^{k}}{1-\left(\mathrm{e}^{-\pi \delta}\right)^{k}}
$$

Inserting Eq.26) and Eq. 30 into Eq. 34 yields the time-domain approach damage:

$$
D_{R}=\left(\frac{A_{c} \mathrm{e}^{-\frac{1}{2}(\pi \delta)}}{C \omega_{0}}\right)^{k}+\frac{\left(\frac{A_{c} \mathrm{e}^{-\frac{1}{2}(3 \pi \delta)} \cosh (\pi \delta / 2)\left(\mathrm{e}^{\pi \delta}+1\right)}{C \omega_{0}}\right)^{k}}{1-\left(\mathrm{e}^{-\pi \delta}\right)^{k}}
$$

In lightly damped structures $\delta<<1$, the $\cosh (\pi \delta / 2) \approx 1$, and Eq. (37) simplifies to:

$$
D_{R}=\left(\frac{A_{c} \mathrm{e}^{-\frac{1}{2}(\pi \delta)}}{C \omega_{0}}\right)^{k}+\frac{\left(\frac{A_{c} \mathrm{e}^{-\frac{1}{2}(3 \pi \delta)}\left(\mathrm{e}^{\pi \delta}+1\right)}{C \omega_{0}}\right)^{k}}{1-\left(\mathrm{e}^{-\pi \delta}\right)^{k}}
$$

which represents the analytical equation for the damage accumulation of a single half-sine 233 impulse exciting a SDOF system.

\subsection{SDOF response in the frequency domain}

The Fourier transform of the half-sine impulse function $f_{s / 2}$ Eq. 223] is [25]:

$$
F_{s / 2}(r)=\frac{A_{f}\left(1+\mathrm{e}^{2 \mathrm{i} \pi^{2} r}\right)}{1-4 \pi^{2} r^{2}}
$$


where $r$ is the frequency ratio $r=\omega / \omega_{0}$ and $A_{f}$ is the maximum amplitude of the impulse spectrum, which depends on the amplitude of the impulse $A$ and the length of the impulse:

$$
A_{f}=\frac{2 A}{a \omega_{0}}
$$

The Fourier transform of the impulse-response function $g(t)(3)$ is:

$$
G(r)=\frac{1 / k_{v}}{-r^{2}+2 \mathrm{i} \delta r+1},
$$

where $k_{v}$ is the stiffness of a SDOF system.

The response PSD is:

$$
S_{x x}(\omega)=(F(\omega) G(\omega))^{*}(F(\omega) G(\omega)),
$$

where the ${ }^{*}$ operator stands for the conjugation of the complex value. The frequency-counting methods used in this paper operate on the spectral moments 14. of the power spectral density of the response.

The PSD $S_{F F}$ is shown in Fig. 3 and we can observe that the excitation PSD is falling at higher frequencies, e.g. when the relative frequency $r$ is higher than 2; this observation will later be used to define the parameter $a$.

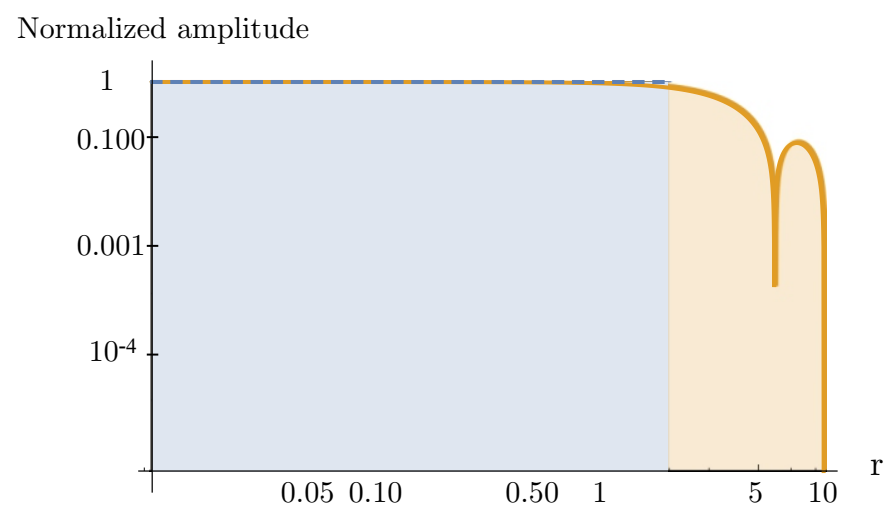

Figure 3: Comparison of the analytical (dashed line) and simplified Fourier transform (full line) of the half-sine impulse.

When multiplied by the impulse transfer function $G(r)$ 41, the response spectrum PSD $S_{x x}$ is less than $1 \%$ of the maximum amplitude for lightly damped structures, Fig. 4. 
The 4-th spectral moment is required to determine $\alpha_{2}$ in the fatigue-damage estimation with the Tovo-Benasciutti method (13). Due to the infinite integral (14) the 4-th moment becomes infinite, which is not reasonable as the system is not excited significantly above $\omega_{0}$, see Fig. 4. For this reason the $F_{s / 2}(39)$ is simplified:

$$
\widetilde{F}_{s / 2}(\omega)=A_{f} \theta\left(a \omega_{0}-\omega\right),
$$

130 $a=2$ is shown in Fig. 3 and 4 .

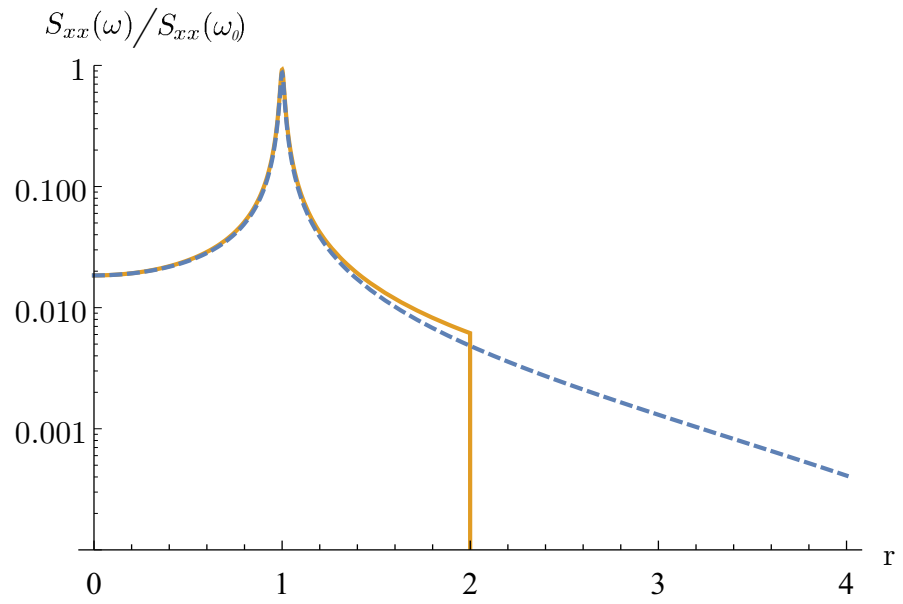

Figure 4: Comparison of the analytical (dashed line) and simplified (full line) response PSD (normalized to $S_{x x}\left(\omega_{0}\right)$ ).

By substituting Eq. (41) into Eq. (43) the spectral moments can be obtained analytically. The first spectral moment is:

$$
\tilde{m}_{0}=\int_{0}^{\infty} \frac{4 A^{2}}{m^{2} a^{2} \omega_{0}^{2}\left(r^{4}+\left(4 \delta^{2}-2\right) r^{2}+1\right)} \mathrm{d} r
$$

which simplifies to:

$$
\tilde{m}_{0}=\frac{A^{2}}{2 m^{2} a^{2}\left(\delta^{3}+\delta\right) \omega_{0}^{5}} .
$$

Assuming lightly damped systems $\delta<<1$, which are common in structural dynamics, the higher-order terms of the damping coefficient can be neglected 
$\delta^{j} \approx 0:$

$$
\tilde{m}_{0} \approx \frac{A^{2}}{2 m^{2} a^{2} \delta \omega_{0}^{5}} .
$$

Similarly, assuming small damping ratios, the next two spectral moments can be approximated as:

$$
\begin{aligned}
& \tilde{m}_{1} \approx \frac{A^{2}\left(\pi-2 \tan ^{-1}(\delta)\right)}{\pi^{2} a^{2} \delta \omega_{0}^{4} m^{2}} \\
& \tilde{m}_{2} \approx \frac{A^{2}}{2 \pi^{2} a^{2} \delta \omega_{0}^{3} m^{2}} .
\end{aligned}
$$

Using Eq. 10 the narrow-band method's damage estimation using the approximated spectral moments is:

$$
D^{N B}=\frac{2^{k-1} \Gamma\left(\frac{k}{2}+1\right) \delta^{-\frac{k}{2}} \omega_{0}^{1-\frac{5 k}{2}}\left(\frac{a m}{A}\right)^{-k}}{\pi C^{k}},
$$

and the fatigue-life estimation is the inverse value of the damage:

$$
L^{N B}=\frac{1}{D^{N B}}
$$

The fourth spectral moment $m_{4}$ is integrated using a finite upper limit of the integration. The upper integration limit $a \omega_{0}$ was used. $a$ should be such that the response PSD of an impulse-excited SDOF structure can be neglected after the upper limit, see Fig. 4 Using the upper integration limit $a \omega_{0}$ and the assumption of a small damping coefficient, the approximated spectral moment $\tilde{m}_{4}$ is:

$$
\tilde{m}_{4} \approx \frac{A^{2}\left(8 \pi a \delta-\mathrm{i}(\delta-\mathrm{i})^{3} \tan ^{-1}\left(\frac{2 \pi a}{\delta-\mathrm{i}}\right)+\mathrm{i}(\delta+\mathrm{i})^{3} \tan ^{-1}\left(\frac{2 \pi a}{\delta+\mathrm{i}}\right)\right)}{8 \pi^{5} a^{2} \delta \omega_{0} m^{2}} .
$$

After applying the assumption of a small damping ratio and using the real part of the equation, $m_{4}$ becomes approximately:

$$
\tilde{m}_{4} \approx \frac{A^{2}\left(-6 \delta^{2} \tan ^{-1}\left(\frac{2 \pi a}{\delta}\right)+8 \pi a \delta+2 \tan ^{-1}\left(\frac{2 \pi a}{\delta}\right)\right)}{8 \pi^{5} a^{2} \delta \omega_{0} m^{2}}
$$

Table 1 shows the relationship between the numerically and analytically obtained approximations of the spectral moments. The numerical moments were obtained by numerically integrating Eq. (14) from 0 to $a \omega_{0}$. For this calculation 
$a=2$ and $\omega_{0}=1$ were used. A similar analysis was performed for the sensitivity of the analytically obtained spectral moments to the natural frequency of the SDOF system in Table 2. Here, the value of the damping ratio was held constant at $\delta=0.01$.

Table 1: Comparison of the numerically and analytically obtained spectral moments $\left(\omega_{0}=1\right)$.

\begin{tabular}{l|llll}
$\delta$ & $m_{0}^{\mathrm{Num}} / \tilde{m}_{0}$ & $m_{1}^{\mathrm{Num}} / \tilde{m}_{1}$ & $m_{2}^{\mathrm{Num}} / \tilde{m}_{2}$ & $m_{4}^{\mathrm{Num}} / \tilde{m}_{4}$ \\
\hline 0.001 & 0.9794 & 0.9791 & 0.9789 & 0.9788 \\
0.01 & 1.0000 & 1.0000 & 1.0010 & 1.0013 \\
0.1 & 1.0000 & 1.0004 & 1.0103 & 1.0060
\end{tabular}

Table 2: Comparison of the numerically and analytically obtained spectral moments $(\delta=$ $0.01)$.

\begin{tabular}{l|llll}
$\omega_{0}$ & $m_{0}^{\mathrm{Num}} / \tilde{m}_{0}$ & $m_{1}^{\mathrm{Num}} / \tilde{m}_{1}$ & $m_{2}^{\mathrm{Num}} / \tilde{m}_{2}$ & $m_{4}^{\mathrm{Num}} / \tilde{m}_{4}$ \\
\hline 10 & 1.0000 & 1.0000 & 1.0010 & 1.0013 \\
100 & 1.0000 & 1.0000 & 1.0010 & 1.0013 \\
1000 & 1.0000 & 1.0000 & 1.0010 & 1.0013
\end{tabular}

Tables 1 and 2 support the simplifications made to obtain the approximations of the spectral moments.

3.3. Comparing the frequency- to time-domain counting methods for half-sine impulse excitation

In order to compare the simplified frequency-domain method Eq. (48) to the time-domain approach, the ratio between the individual life-estimation methods is defined:

$$
R^{N B}=\frac{D^{N B}}{D^{R F}}=\frac{2^{k-1} \Gamma\left(\frac{k}{2}+1\right) \delta^{-\frac{k}{2}} \omega_{0}^{1-\frac{5 k}{2}}\left(\frac{a m}{A_{f}}\right)^{-k}}{\pi\left(\left(A_{c} e^{-\frac{1}{2}(\pi \delta)}\right)^{k}+\frac{\left(A_{c} e^{-\frac{1}{2}(3 \pi \delta)}\left(e^{\pi \delta}+1\right)\right)^{k}}{1-\left(e^{-\pi \delta}\right)^{k}}\right)},
$$

where $D^{N B} 48$ is the narrow-band method and $D^{R F}(38)$ the rainflow method, see Fig. 5 
Similarly, the ratio can be defined for the Tovo-Bennasciutti method as:

$$
R^{T B}=\frac{D^{T B}}{D^{R F}} .
$$

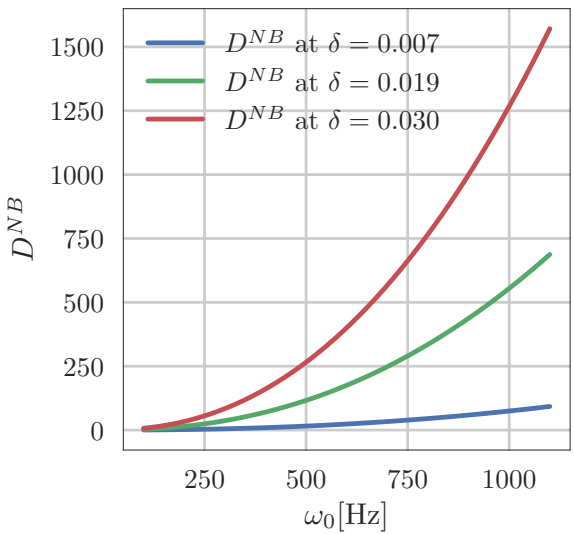

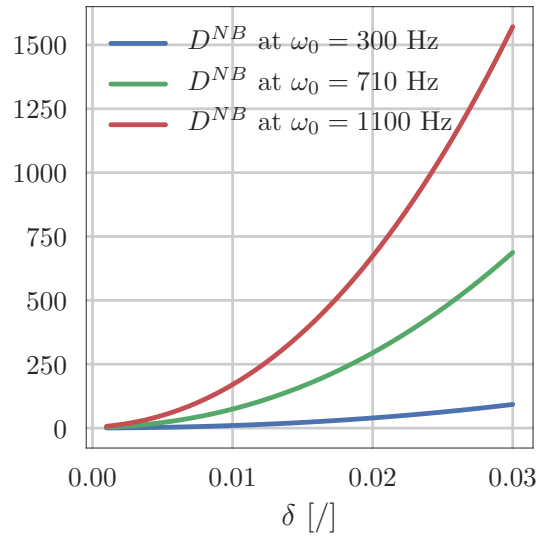

Figure 5: $R^{N B}$ with fixed material parameters.

. It is clear from equation (52) that the theoretical time-to-failure prediction discrepancies between the time- and frequency-domain approaches vary significantly with the dynamic properties of the structure.

\section{Experiment}

\subsection{Experimental setup}

To support the theoretical approach an experimental setup was prepared. A standard Y sample was chosen [39, 31. In general Y-shape sample behaves as a multi-degree of freedom system. However, as the dynamic modes of the 
sample are well separated, the sample can be approximated as a SDOF system, weights, and the stiffness $k$ is the stiffness of the sample arms. 


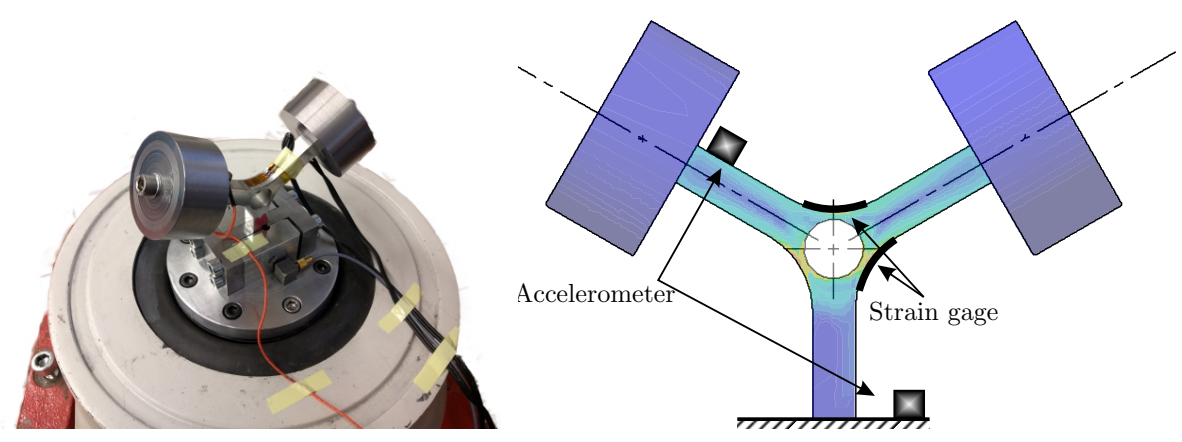

Figure 6: Experimental setup.

The modal decomposition of the system in Fig 7 to two separate degrees of freedom is required to use the derived equation. This is presented in detail in 190 Appendix B

The relationship between the stresses at the measured points and the relative displacements $\left(k_{\sigma}\right)$ was then determined, similar to the procedure described in [31.

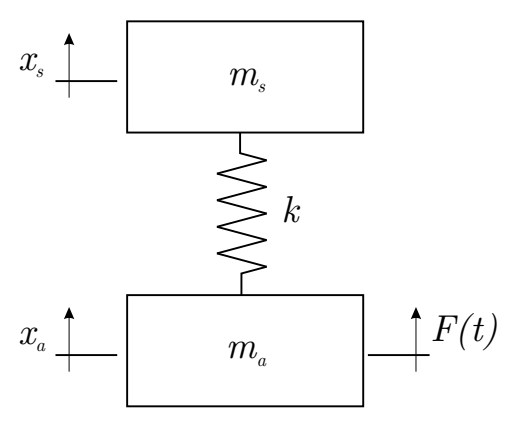

Figure 7: Kinematic 2 DOF model of the experiment.

The frequency of the shocks was set to 2 shocks per second. It was ensured 195 that the response of the sample was below $0.1 \%$ of the peak response before the start of the next impulse. The duration of the impulses was chosen so that the PSD of the impulse was close to linear near the natural frequency of the system [21]. During the testing the value of $a=2$ was used to determine the 
duration of the impulse and consequently ensured that the natural dynamics of the selected mode of the dynamic system was well excited, see Fig. 7 . The frequency response of the sample was well within the linear range. Each sample was excited with a constant amplitude of the impulse.

\subsection{Data analysis}

Due to the long-lasting experiments (the samples at the low excitation level were tested for several weeks), only 3 sets of 3 samples were tested in order to support the theoretical analysis. Different weights were used for each set in order to vary the natural frequency and damping of the samples, see Tab. 3 The damping coefficient was determined using the time-domain logarithmic decrament model [40]:

$$
\delta=\frac{1}{n} \ln \frac{x(t)}{x\left(t+n T_{0}\right)},
$$

where $T_{0}=1 / \omega_{0}$. The factor $a$ (24) that determines the length of the impulse was held constant for all the samples, with a value of $a=2$.

Table 3: Identified modal parameters of the samples.

\begin{tabular}{c|ccc} 
Sample nr. & Frequency $[\mathrm{Hz}]$ & Damping coefficient $[/]$ & Peak impulse stress $[\mathrm{MPa}]$ \\
\hline 1.1 & 300.0 & 0.0269 & 209.94 \\
1.2 & 300.0 & 0.0240 & 198.58 \\
1.3 & 300.0 & 0.0266 & 209.76 \\
\hline 2.1 & 330.0 & 0.0164 & 167.35 \\
2.2 & 334.0 & 0.0185 & 152.92 \\
2.3 & 328.0 & 0.0165 & 158.58 \\
3.1 & 402.0 & 0.0148 & 113.68 \\
3.2 & 398.5 & 0.0108 & 120.48 \\
3.3 & 402.0 & 0.0202 & 127.86
\end{tabular}

\subsubsection{Fatigue parameters}

The structural dynamics changes far before the complete failure of the sample; therefore it is practical to identify failure when the natural frequency starts 
to change rapidly. In this research the natural frequency drop by $2 \%$ was considered failure. For details on the failure on damage identification from natural frequency, the interested reader is refered to: 34, 41. Fig. 8 shows a histogram of the rainflow cycles of a selected sample. The fatigue parameters were determined by a least-squares optimization by relating the numerical time-domain rainflow to the experimentally identified damage.

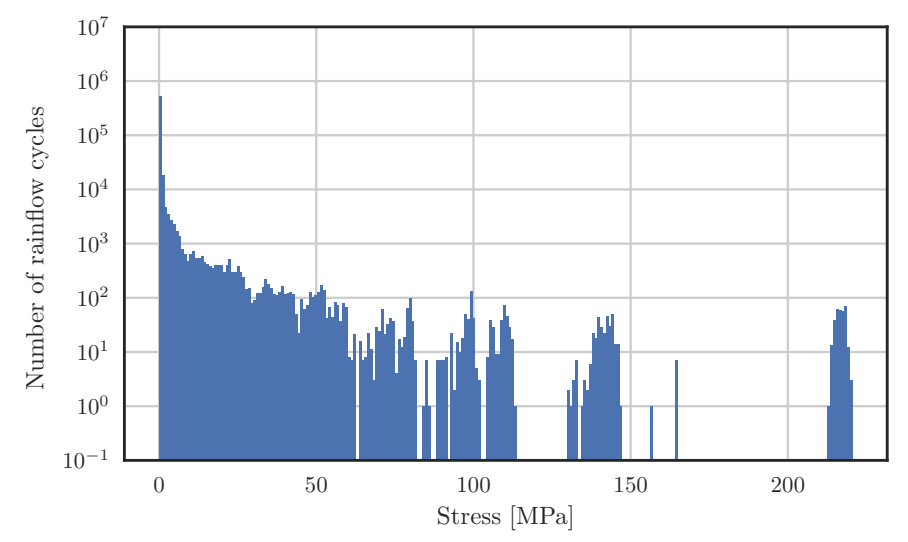

Figure 8: Histogram of the rainflow cycles for the sample 1.3.

The following fatigue parameters were identified:

$$
\begin{aligned}
k & =-6.51 \\
C & =800.26 .
\end{aligned}
$$

215 These fatigue parameters were then used to determine the estimated fatigue life $L^{R F}=1 / D^{R F}$ (38), see Tab. 4

\subsubsection{The frequency-domain life estimations}

Based on the material's properties, the natural frequency, the damping and the excitation pulse parameters, the fatigue life $L^{N B} 49$ can be estimated, see Tab. 4. Additionally, the ratio $R^{N B} \sqrt{52}$ is given. If the fatigue-life estimation $L^{N B}$ is corrected for the ratio $R^{N B}$ the corrected $L_{\text {corr }}^{N B}$ can be obtained. 
Table 4: Fatigue-life estimates for the rainflow and the narrow-band methods.

\begin{tabular}{c|ccccc} 
Sample nr. & $\begin{array}{c}\text { Experiment } \\
{[\mathrm{s}]}\end{array}$ & $L^{R F}$ & $L^{N B}$ & $R^{N B}$ & $L_{\text {corr }}^{N B}$ \\
& 3650 & 5305 & 627964 & 130.60 & 4808 \\
1.1 & 19460 & 12327 & 1655389 & 107.23 & 15438 \\
1.2 & 3230 & 8734 & 1180594 & 128.13 & 9214 \\
\hline 1.3 & 52780 & 20029 & 770556 & 34.84 & 22120 \\
\hline 2.1 & 113090 & 32106 & 1232668 & 44.98 & 27403 \\
2.2 & 116540 & 23156 & 799102 & 34.76 & 22987 \\
\hline 2.3 & 154830 & 62583 & 1776052 & 29.48 & 60242 \\
3.1 & 66090 & 45511 & 1274479 & 15.41 & 82660 \\
3.3 & 103070 & 46319 & 1245921 & 53.20 & 23419
\end{tabular}

The results of the experimental analysis is presented in Fig. 9. The Figure presents the relations between the fatigue life estimation obtained by the narrowband and rainflow method. The life estimations of the narrow-band method were 225 corrected with the analytically obtained ratio $R^{N B}$. This moves the frequencydomain narrow-band method life estimation closer to the time-domain rainflow and experimental life. 


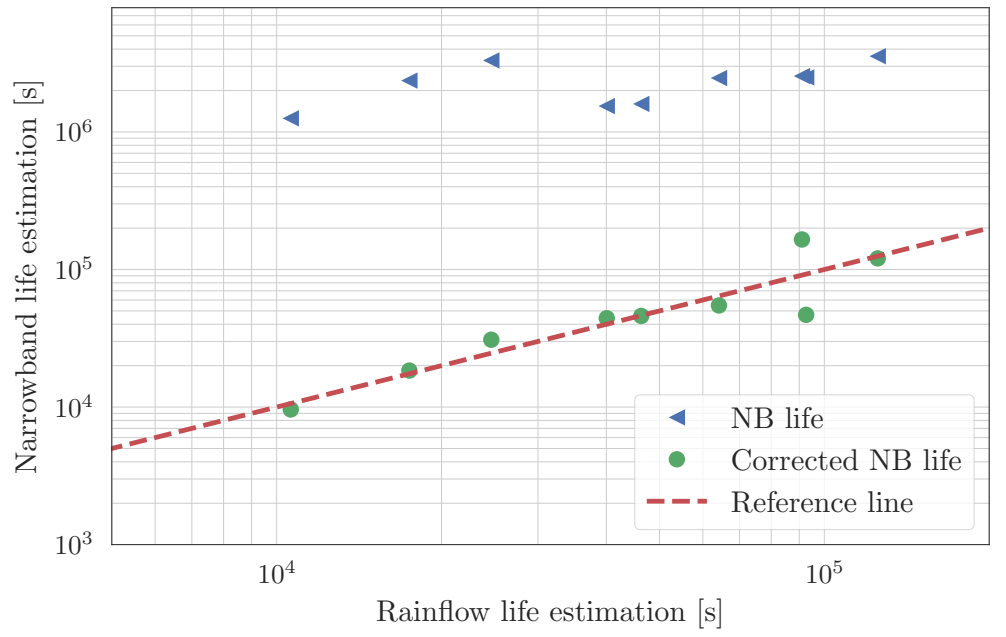

Figure 9: Comparison of the narrow-band and corrected narrow-band life estimation with regards to the experimental rainflow fatigue lives.

Similarly, the results for the Tovo-Benasciutti method the ratio $R^{T B}$ was determined, and the life estimations $L^{R F}$ and $L^{T B}$, together with the corrected ${ }_{230}$ life estimation $L_{\text {corr }}^{T B}$, are presented in Table 5

Table 5: Fatigue-life estimations for the rainflow and the Tovo-Benasciutti method.

\begin{tabular}{c|ccccc} 
Sample nr. & $\begin{array}{c}\text { Experiment } \\
{[\mathrm{s}]}\end{array}$ & $\begin{array}{c}L^{R F} \\
{[\mathrm{~s}]}\end{array}$ & $\begin{array}{c}L^{T B} \\
{[\mathrm{~s}]}\end{array}$ & $\begin{array}{c}R^{T B} \\
{[/]}\end{array}$ & $\begin{array}{c}L_{\text {corr }}^{T B} \\
{[\mathrm{~s}]}\end{array}$ \\
\hline 1.1 & 3650 & 5305 & 627964 & 102.51 & 6126 \\
1.2 & 19460 & 12327 & 1655389 & 85.70 & 19315 \\
1.3 & 3230 & 8734 & 1180594 & 100.75 & 11718 \\
\hline 2.1 & 52780 & 20029 & 770556 & 34.84 & 22120 \\
2.2 & 113090 & 32106 & 1232668 & 37.33 & 33023 \\
2.3 & 116540 & 23156 & 799102 & 29.28 & 27291 \\
\hline 3.1 & 154830 & 62583 & 1776052 & 25.16 & 70575 \\
3.2 & 66090 & 45511 & 1274479 & 13.62 & 93585 \\
3.3 & 103070 & 46319 & 1245921 & 43.62 & 28565
\end{tabular}

The comparison for the Tovo-Bennasciutti method and the rainflow method, 
with the corrected Tovo-Bennasciutti method, is presented in Fig. 10

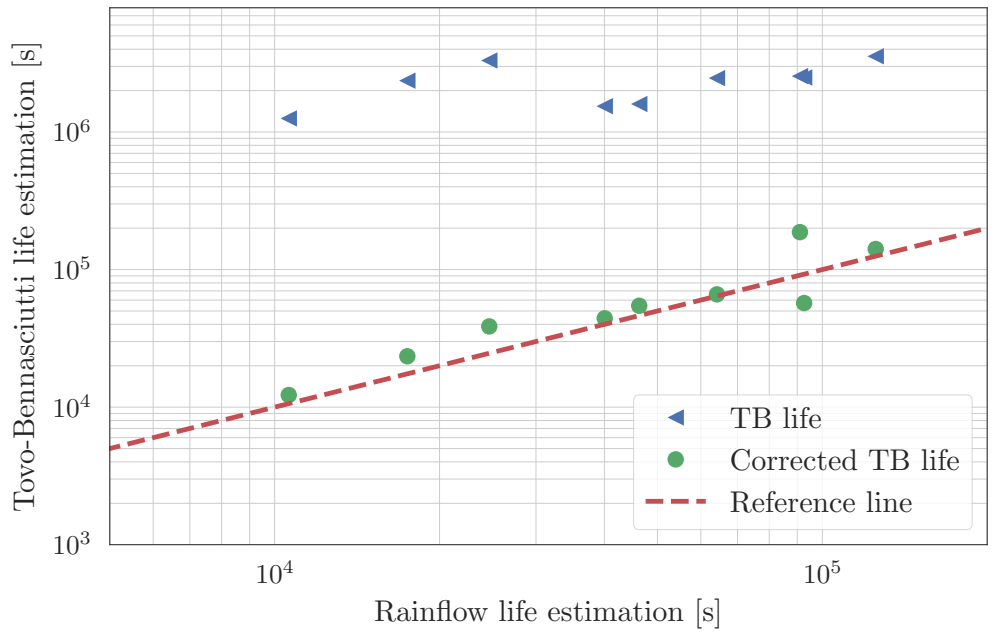

Figure 10: Comparison of the Tovo-Bennasciutti and corrected Tovo-Bennasciutti life estimation with regards to the experimental rainflow fatigue lives.

In the Tables 4 and 5 discrepancies between the calculated rainflow lives and the actual experimental fatigue lives are noticeable. These are the cause of the variation between the individual samples.

With the proposed model it was possible to significantly improve the fatigue life predictions for both the narrow-band and the Tovo-Bennaciutti method. While the analysed process is distinctly narrow-banded, the derivation for the TovoBennasciutti method was also carried out, as the Tovo-Bennasciutti method can be applied also to a broadband band process.

\section{Conclusion}

This theoretical and experimental study focuses on the discrepancies between the time-domain and frequency-domain fatigue-life estimations when dealing with impulse-excitation loads. As the impulses render the signal non-stationary and non-Gaussian the accuracy of the frequency methods becomes questionable. Even for structures that are intended to be exposed to random loads only, the impulse loading can appear due to clearances. 
As can be seen in Tables 5 and 4 the frequency-domain methods predict significantly longer fatigue lives when compared to the experimentally measured time-domain observations. This difference originates in the basic inability of the frequency-domain methods to analyse non-stationary and non-Gaussian processes. It was further found that this difference depends on the dynamic properties of the system (i.e., natural frequency, damping), the impulse-excitation parameters and the fatigue material parameters.

255

This manuscript introduces a theoretically deduced ratio between the timeand frequency-domain methods (narrow-band and Tovo-Benasciutti). The proposed solution takes into account the dynamic properties, the impulse excitation and the fatigue parameter, and was shown to give accurate results also in the frequency domain, assuming linear relations. The proposed correction was also experimentally confirmed.

\section{Acknowledgment}

The authors acknowledge the partial financial support from the Slovenian Research Agency (research core funding No. P2-0263).

\section{References}

265 [1] D. E. Newland, An introduction to random vibrations, spectral \& wavelet analysis, Courier Corporation, 2012.

[2] F. Cianetti, A. Alvino, A. Bolognini, M. Palmieri, C. Braccesi, The design of durability tests by fatigue damage spectrum approach, Fatigue \& Fracture of Engineering Materials \& Structures 41 (4) (2018) 787-796.

270

[3] Y. Kong, S. Abdullah, D. Schramm, M. Omar, S. Haris, Vibration fatigue analysis of carbon steel coil spring under various road excitations, Metals 8 (8) (2018) 617. 
[4] M. Mršnik, J. Slavič, M. Boltežar, Frequency-domain methods for a vibration-fatigue-life estimation-application to real data, International Journal of Fatigue 47 (2013) 8-17.

[5] A. Angeli, B. Cornelis, M. Troncossi, Synthesis of sine-on-random vibration profiles for accelerated life tests based on fatigue damage spectrum equivalence, Mechanical Systems and Signal Processing 103 (2018) 340-351.

[6] A. Carpinteri, A. Spagnoli, S. Vantadori, A review of multiaxial fatigue criteria for random variable amplitude loads, Fatigue \& Fracture of Engineering Materials \& Structures 40 (7) (2017) 1007-1036.

[7] D. Zanellati, D. Benasciutti, R. Tovo, Vibration fatigue tests by tri-axis shaker: design of an innovative system for uncoupled bending/torsion loading, Procedia Structural Integrity 8 (2018) 92-101.

${ }_{285}$ [8] M. Palmieri, M. Česnik, J. Slavič, F. Cianetti, M. Boltežar, Non-gaussianity and non-stationarity in vibration fatigue, International Journal of Fatigue 97 (2017) 9-19.

[9] M. Matsuishi, T. Endo, Fatigue of metals subjected to varying stress, Japan Society of Mechanical Engineers, Fukuoka, Japan 68 (2) (1968) 37-40.

[10] J. W. Miles, On structural fatigue under random loading, Journal of the Aeronautical Sciences 21 (11) (1954) 753-762.

[11] T. Dirlik, Application of computers in fatigue analysis, Ph.D. thesis, University of Warwick (1985).

[12] D. Benasciutti, R. Tovo, Frequency-based fatigue analysis of non-stationary switching random loads, Fatigue \& Fracture of Engineering Materials \& Structures 30 (11) (2007) 1016-1029.

[13] J. S. Bendat, A. G. Piersol, Random data: analysis and measurement procedures, Vol. 729, John Wiley \& Sons, 2011. 
[14] M. Böhm, T. Eagoda, Fatigue life assessment with the use of the spectral method for non-gaussian loading histories with the use of the energy parameter, Journal of Machine Construction and Maintenance. Problemy Eksploatacji 01 (2018) 27-31.

[15] Z. Fan, Y. Jiang, S. Zhang, J. Tao, X. Chen, Research on vibration fatigue of carbon fibre-reinforced composites under non-gaussian random load, in: Prognostics and System Health Management Conference (PHM-Harbin), 2017, IEEE, 2017, pp. 1-8.

[16] F. Cianetti, M. Palmieri, C. Braccesi, G. Morettini, Correction formula approach to evaluate fatigue damage induced by non-gaussian stress state, Procedia Structural Integrity 8 (2018) 390-398.

[17] P. Wolfsteiner, W. Breuer, Fatigue assessment of vibrating rail vehicle bogie components under non-gaussian random excitations using power spectral densities, Journal of Sound and Vibration 332 (22) (2013) 5867-5882.

[18] P. Wolfsteiner, S. Sedlmair, Deriving gaussian fatigue test spectra from measured non gaussian service spectra, Procedia Engineering 101 (2015) $543-551$.

[19] P. Wolfsteiner, Fatigue assessment of non-stationary random vibrations by using decomposition in gaussian portions, International Journal of Mechanical Sciences 127 (2017) 10-22.

[20] Q. Niu, S.-x. Yang, X.-l. Li, An empirical mode decomposition-based frequency-domain approach for the fatigue analysis of nonstationary processes, Fatigue \& Fracture of Engineering Materials \& Structures (2018) $1-17$.

[21] S. A. Rizzi, A. Przekop, T. L. Turner, On the response of a nonlinear structure to high kurtosis non-gaussian random loadings, in: EURODYN20118th International Conference on Structural Dynamics; (2011). 
[22] X. Song, S. Wang, A novel spectral moments equivalence based lumping block method for efficient estimation of offshore structural fatigue damage, International Journal of Fatigue 118 (2019) 162-175.

[23] L. Capponi, M. Česnik, J. Slavič, F. Cianetti, M. Boltežar, Non-stationarity index in vibration fatigue: Theoretical and experimental research, International Journal of Fatigue 104 (2017) 221-230.

[24] D. Benasciutti, Fatigue analysis of random loadings, PhD University of Ferrara, Department of Engineering, (2004).

[25] N. M. M. Maia, J. M. M. e Silva, Theoretical and experimental modal analysis, Research Studies Press, 1997.

[26] M. Géradin, D. J. Rixen, Mechanical vibrations: theory and application to structural dynamics, John Wiley \& Sons, 2014.

[27] S. W. Smith, et al., The scientist and engineer's guide to digital signal processing, California Technical Pub. San Diego, 1997.

[28] K. Shin, J. K. Hammond, Fundamentals of signal processing for sound and vibration engineers, John Wiley \& Sons, 2008.

[29] D. F. Socie, G. B. Marquis, Multiaxial fatigue, Society of Automotive Engineers Warrendale, PA, 2000.

[30] A. Wöhler, Über die Festigkeits-versuche mit Eisen und Stahl, 1870.

[31] M. Česnik, J. Slavič, M. Boltežar, Uninterrupted and accelerated vibrational fatigue testing with simultaneous monitoring of the natural frequency and damping, Journal of Sound and Vibration 331 (24) (2012) 5370-5382.

[32] D. Benasciutti, R. Tovo, Frequency-based analysis of random fatigue loads: Models, hypotheses, reality: Frequenzbasierte analyse zufälliger ermüdungsbelastungen: Modelle, hypothesen, praxis, Materialwissenschaft und Werkstofftechnik 49 (3) (2018) 345-367. 
[33] R. Blevins, Non-gaussian narrow-band random fatigue, Journal of applied mechanics 69 (3) (2002) 317-324.

[34] M. Mršnik, J. Slavič, M. Boltežar, Multiaxial vibration fatigue; a theoretical and experimental comparison, Mechanical Systems and Signal Processing 76 (2016) 409-423.

[35] A. Nieslony, E. Macha, Spectral method in multiaxial random fatigue, Vol. 33, Springer Science \& Business Media, 2007.

[36] K. G. McConnell, Vibration testing: theory and practice, John Wiley \& Sons, 1995.

[37] C. Lalanne, Mechanical vibration and shock analysis, fatigue damage, Vol. 4, John Wiley \& Sons, 2010.

[38] W. Rudin, et al., Principles of mathematical analysis, Vol. 3, McGraw-hill New York, 1976.

[39] M. Mršnik, J. Slavič, M. Boltežar, Vibration fatigue using modal decomposition, Mechanical Systems and Signal Processing 98 (2018) 548-556.

[40] D. J. Inman, R. C. Singh, Engineering vibration, Vol. 3, Prentice Hall Englewood Cliffs, NJ, 1994.

[41] J.-T. Kim, Y.-S. Ryu, H.-M. Cho, N. Stubbs, Damage identification in beam-type structures: frequency-based method vs mode-shape-based method, Engineering structures 25 (1) (2003) 57-67.

[42] S. S. Rao, F. F. Yap, Mechanical vibrations, Vol. 4, Prentice Hall Upper Saddle River, 2011.

\section{Appendix A. Structural dynamics}

The equation of motion for a general multiple-degree-of-freedom (MDOF) structure is [26, 42]:

$$
\mathbf{M} \ddot{\mathbf{x}}+\mathbf{C} \dot{\mathbf{x}}+\mathbf{K} \mathbf{x}=\mathbf{f}
$$


where $\mathbf{M}, \mathbf{C}$ and $\mathbf{K}$ are the mass, viscous damping and stiffness matrices of the structure, respectively. $\mathbf{f}$ represents the vector of the excitation forces and $\mathbf{x}$ is the displacement vector of the stucture's degrees of freedom. After introducing the proportional viscous damping and modal coordinates, the equations of motion become uncoupled [25].

$$
\mathbf{I} \ddot{\mathbf{q}}+\left[\backslash \mathbf{2} \xi \omega_{\mathbf{0}} \backslash\right] \dot{\mathbf{q}}+\left[\backslash \omega_{\mathbf{0}}^{2} \backslash \mathbf{q}=\boldsymbol{\Phi}^{T} \mathbf{F}(\omega),\right.
$$

where:

$$
\mathbf{x}=\mathbf{\Phi} \mathbf{q}
$$

$\mathbf{q}$ are the modal coordinates and $\mathbf{\Phi}$ is the mass-normalized modal matrix [25]. I represents the identity matrix, $\left[\backslash \mathbf{2} \xi \omega_{\mathbf{0}} \backslash\right]$ is the diagonal damping matrix and $\left[\backslash \omega_{0}^{2} \backslash\right]$ is the diagonal matrix of natural frequencies. $\omega_{0}$ stands for the natural frequency of the uncoupled modal degrees of freedom and $\xi$ is the corresponding coefficient of viscous damping. For the $i$-th modal coordinate $q_{i}$ the equation of motion is [25]:

$$
\ddot{q}_{i}+2 \xi_{i} \omega_{0, i} \dot{q}_{i}+\omega_{0, i}^{2} q_{i}=\Phi_{i}^{T} F(\omega)
$$

where $\Phi_{i}$ is the mass-normalized eigenvector of the $i$-th degree of freedom.

\section{Appendix B. Modal decomposition of the 2 DOF system}

The equation of motion for the system in Fig. 7(Eq. A.1) is:

$$
\left[\begin{array}{cc}
m_{s} & 0 \\
0 & m_{a}
\end{array}\right]\left(\begin{array}{l}
\ddot{x}_{s} \\
\ddot{x}_{a}
\end{array}\right)+\left[\begin{array}{cc}
k & -k \\
-k & k
\end{array}\right]\left(\begin{array}{l}
x_{s} \\
x_{a}
\end{array}\right)=\left(\begin{array}{c}
0 \\
F(t)
\end{array}\right)
$$

After solving Eq. (B.1) for its eigenvalues, the natural frequencies are:

$$
\omega_{0,1}=0, \quad \omega_{0,2}=\frac{\sqrt{k} \sqrt{m_{s}+m_{a}}}{\sqrt{m_{s}} \sqrt{m_{a}}}
$$

and the modal matrix:

$$
\mathbf{\Phi}=\left(\begin{array}{cc}
1 & -\frac{m_{a}}{m_{s}} \\
1 & 1
\end{array}\right)
$$


Using the modal coordiantes A.2.

$$
\left[\begin{array}{ll}
1 & 0 \\
0 & 1
\end{array}\right]\left(\begin{array}{c}
\ddot{q}_{1} \\
\ddot{q}_{2}
\end{array}\right)+\left[\begin{array}{cc}
\omega_{0,1}^{2} & 0 \\
0 & \omega_{0,2}^{2}
\end{array}\right]\left(\begin{array}{l}
q_{1} \\
q_{2}
\end{array}\right)=\left(\begin{array}{c}
F(t) \\
F(t)
\end{array}\right)
$$

And finally, the motion equation of the flexible (non-zero natural frequency) mode shape is:

$$
\ddot{q}_{2}+2 \xi_{2} \omega_{0,2} \dot{q}_{2}+\omega_{0,2}^{2} q_{2}=F(t)
$$

Using Eq. A.3 the physical coordinates $\mathbf{x}$ are:

$$
\left(\begin{array}{c}
x_{s} \\
x_{a}
\end{array}\right)=\mathbf{\Phi} \mathbf{q}=\left(\begin{array}{c}
q_{1}-\frac{m_{a}}{m_{s}} q_{2} \\
q_{1}+q_{2}
\end{array}\right)
$$

The relative displacement between the sample and shaker armature is defined:

$$
\Delta x=x_{s}-x_{a}=-q_{2}-\frac{m_{a}}{m_{s}} q_{2}
$$

and can, under a linear assumption, be related to the stress response 222. Eq. 34 and (48) can then be used on the $q_{2}$ degree of freedom to determine the damage to the sample. 